\title{
A RATIONAL TORSION INVARIANT
}

\author{
JOHN EWING, PETER LÖFFLER AND ERIK KJAER PEDERSEN
}

(Communicated by Haynes R. Miller)

\begin{abstract}
We show that for spaces with rational cohomology an exterior algebra on odd dimensional generators, one can define a torsion invariant which is a rational number. This may be interpreted as an absolute version of the multiplicative Euler characteristic associated to a rational homotopy equivalence.
\end{abstract}

0. Introduction. Reidemeister torsion of a finite complex $X$, with trivial action of the fundamental group on rational homology, takes values in

$$
K_{1}\left(\mathbf{Q} \pi_{1}(X) / \Sigma\right) \subseteq K_{1}\left(\mathbf{Q} \pi_{1}(X)\right)
$$

where $\Sigma=\sum_{g \in \pi_{1}(X)} g$. It is the purpose of this paper to show that one can sometimes define a "Reidemeister torsion" invariant in the other factor of $K_{1}\left(\mathbf{Q} \pi_{1}(X)\right)=$ $K_{1}\left(\mathbf{Q} \pi_{1}(X) / \Sigma\right) \times K_{1}(\mathbf{Q})$.

Specifically, when a space with finitely generated integral homology has rational cohomology an exterior algebra on odd dimensional generators, then we show that there is a torsion invariant defined in $K_{1}(\mathbf{Q}) / K_{1}(\mathbf{Z})=\mathbf{Q}_{+}^{*}$. This may be interpreted as an absolute version of the multiplicative Euler characteristic of a rational homotopy equivalence that has been utilized by a number of authors (J. Davis, P. Löffler, G. Mislin, and S. Weinberger).

In case the space $X$ is also nilpotent with finite fundamental group, we show that this invariant is an obstruction to a free $S^{1}$ action on the homotopy type of $X$ having finitely dominated quotient. We also show that the invariant can be used to extend the definition of linking number of odd dimensional spheres to rational homology spheres.

1. Algebraic preliminaries. Consider an exterior algebra over $\mathbf{Q}, \boldsymbol{\Lambda}=$ $\Lambda\left[x_{1}, x_{2}, \ldots, x_{n}\right]$ where $\operatorname{deg} x_{i}$ is odd. We are interested in the torsions of algebraic isomorphisms of $\Lambda$.

THEOREM 1.1. If $n>1$ then any algebra automorphism of $\Lambda$ has trivial torsion in $K_{1}(\mathbf{Q})$.

For the proof we need a lemma.

An algebra automorphism is given by its values on the generators $x_{i}$. We say an automorphism is elementary if it is the identity on all but one of the $x_{i}$, and the one exceptional $x_{i}$ is mapped to $x_{i}$ +monomial, where the monomial is not a multiple of $x_{i}$.

Received by the editors November 6, 1986.

1980 Mathematics Subject Classification (1985 Revision). Primary 57Q10; Secondary 55P62, $57 \mathrm{Q12}$.

Key words and phrases. Reidemeister torsion, rational homotopy equivalence. 


\section{LEMMA 1.2. The torsion of an elementary automorphism is trivial.}

PROOF. Suppose the elementary automorphism $\lambda$ is the identity except on $x_{i}$, and let $\operatorname{deg}\left(x_{i}\right)=k$. In degrees lower than $k, \lambda$ is the identity. In degree $k, \lambda$ is a usual elementary automorphism of a vector space.

Now suppose $\lambda\left(x_{i}\right)=x_{i}+m$, where $m$ is the monomial. In degrees higher than $k$, write $\Lambda_{j}=V \oplus W$, where $W$ is generated as a vector space by monomials that are divisible by $x_{i}$ but do not have a common factor with the monomial $m$, and $V$ is generated by all other monomials. The automorphism $\lambda$ is the identity on $V$, since for each monomial either the monomial does not involve $x_{i}$ or $m$ times the monomial is zero. On $W$ any monomial $\mu$ is sent to $\mu+\left(\mu / x_{i}\right) \cdot m$, showing that $\lambda$ is the product of usual elementary automorphisms of a vector space. Hence, in each degree, $\lambda$ is elementary. It follows that the torsion of $\lambda$ is trivial.

PROOF OF THEOREM 1.1. Let $\Phi$ be an algebra automorphism of $\Lambda$. If $\Phi$ is a Hopf algebra automorphism (that is, $\Phi\left(x_{i}\right)=\alpha_{i} x_{i}$ ) the result is easy to show, and has been exploited by Mislin [ M2, p. 555].

We shall now convert $\Phi$ to a Hopf algebra automorphism by composition with elementary automorphisms. In the lowest dimension we can clearly change $\Phi$ to be diagonal by composition with elementary automorphisms. We then proceed by induction on the degrees of the $x_{i}$. Suppose $x_{i}$ is not sent to a multiple of itself, and all $x_{j}$ in smaller degrees are. Then $x_{i}$ must be sent to

$$
\sum s_{k} x_{k}+\sum t_{j} m_{j}
$$

where $s_{k}$ and $t_{j}$ are rational numbers and the $m_{j}$ are monomials, which are products of elements of lower degree. Composing with the elementary automorphisms that send $x_{i}$ to $x_{i}-t_{j} m_{j}$, we see that $x_{i}$ is now sent to $\sum s_{k} x_{k}$. This, however, is the usual linear situation which can always be diagonalized by composition with elementary automorphisms.

Since elementary automorphisms have trivial torsion by Lemma 1.2, we conclude that $\Phi$ itself has trivial torsion.

2. Definition of the invariant. Let $X$ be a space of finite type (that is, $H_{*}(X)$ is finitely generated) and suppose $H^{*}(X ; \mathbf{Q})$ is an exterior algebra on odd dimensional generators. We can now define the invariant in $K_{1}(\mathbf{Q}) / K_{1}(\mathbf{Z})=\mathbf{Q}_{+}^{*}$ mentioned in the introduction.

We begin by choosing a finitely generated free chain complex $S_{*}$ which is homotopy equivalent to the singular chains of $X$. In $H_{*}\left(S_{*} ; \mathbf{Q}\right)=H_{*}(X ; \mathbf{Q})$, choose a basis dual to the basis for $H^{*}\left(S_{*} ; \mathbf{Q}\right)$ given by monomials. By Theorem 1.1 , this gives a well-defined basis for $H_{*}\left(S_{*} ; \mathbf{Q}\right)$ up to simple isomorphism (when measured in $\mathbf{Q}^{*}$ ).

Now in $S_{*}$ we may choose an integral basis which then induces a basis for $S_{*} \otimes \mathbf{Q}$. Since the chain comblex is based, and the homology is based as well, we obtain as usual a torsion element $q(X) \in K_{1}(\mathbf{Q})=\mathbf{Q}^{*}$. A different choice of integral basis will vary $q(X)$ by an element in the image of $K_{1}(\mathbf{Z}) \rightarrow K_{1}(\mathbf{Q})$. The invariant $q(X)$ is therefore well defined in $K_{1}(\mathbf{Q}) / K_{1}(\mathbf{Z})=\mathbf{Q}_{+}^{*}$.

In case $X$ is also nilpotent with finite fundamental group $\pi$, there is another quite geometric way to describe the invariant $q(X)$. We describe this below. 
Suppose $H^{*}(X ; \mathbf{Q})=\Lambda\left[x_{1}, \ldots, x_{n}\right]$ with $\operatorname{degree}\left(x_{i}\right)=2 k_{i}+1$. By obstruction theory we obtain a homotopy equivalence

$$
X_{(0)} \rightarrow \prod_{i=1}^{n} K\left(\mathbf{Q}, 2 k_{i}+1\right)=\prod_{i=1}^{n} S_{(0)}^{2 k_{i}+1}
$$

Also by obstruction theory, applied to the diagram

$$
\begin{gathered}
X \\
\prod_{i=1}^{n} S^{2 k_{i}+1} \rightarrow \stackrel{\downarrow}{X} \\
(0)
\end{gathered}
$$

one finds a map $\prod_{i=1}^{n} S^{2 k_{i}+1} \rightarrow X$ which induces a rational homotopy equivalence. (To see this, note that any obstruction will have finite order and by composition with a rational equivalence of the product of spheres can be made to vanish.)

The map $\prod_{i=1}^{n} S^{2 k_{i}+1} \rightarrow X$ now induces a map of chain complexes

$$
\Lambda\left[x_{1}, x_{2}, \ldots, x_{n}\right] \rightarrow S_{*},
$$

which is a homotopy equivalence after tensoring with $\mathbf{Q}$. The basis in the homology of $\prod S^{2 k_{i}+1}$ is exactly sent to a basis which is the dual of the monomial basis in $S_{*} \otimes \mathbf{Q}$. Hence, the invariant $q(X)$ can be interpreted as the torsion of this rational homotopy equivalence.

This naturally leads to the multiplicative Euler characteristic, yet another way to define $q(X)$.

DEFINITION 2.1. Let $f: X \rightarrow Y$ be a rational homotopy equivalence of spaces of finite type. The multiplicative Euler characteristic of $f$ is defined to be

$$
m \chi(f)=\prod \frac{\left|H_{\text {odd }}(f)\right|}{\left|H_{\text {even }}(f)\right|} .
$$

LEMMA 2.2. Let $f: X \rightarrow Y$ be a rational homotopy equivalence of finitely dominated spaces. Let $\gamma \in \mathbf{Q}_{+}^{*}$ denote the torsion of the homotopy equivalence. Then $\gamma=m \chi(f)$.

PROOF. Let $S_{*}$ and $T_{*}$ be finitely generated free chain complexes representing the singular chains of $X$ and $Y$ respectively. Let $f_{*}: S_{*} \rightarrow T_{*}$ be a chain map representing $f$. To compute the torsion of $f$, one chooses bases for $S_{*}$ and $T_{*}$, and then notes that the mapping cone of $f_{*}$ is acyclic after tensoring with $\mathbf{Q}$. But the homology of $f$ is by definition the homology of the mapping cone.

Such an integral chain complex is the sum of chain complexes of type

$$
0 \rightarrow \mathbf{Z} \stackrel{n}{\rightarrow} \mathbf{Z} \rightarrow 0
$$

where $n \neq 0$, since the chain complex is $\mathbf{Q}$-acyclic. But the torsion of this short chain complex is simply $n$ or $1 / n$ (depending on the parity). This is, however, exactly the order of the homology.

REMARK 2.3. The interpretation of the multiplicative Euler characteristic as the torsion of the rational homotopy equivalence makes certain properties self-evident. For example, for the composition of rational homotopy equivalences

$$
X \stackrel{f}{\rightarrow} Y \stackrel{g}{\rightarrow} Z
$$

it is now clear that $m \chi(g \cdot f)=m \chi(g) \cdot m \chi(f)$. 
3. Obstructions to $S^{1}$ actions. Suppose $X$ is a nilpotent complex of finite type with finite fundamental group, and suppose the rational cohomology of $X$ is an exterior algebra on odd degree generators. The invariant $q(X)$ is then defined and we have shown in the preceding section that it can be interpreted as the torsion (or multiplicative Euler characteristic) of a rational homotopy equivalence from a product of spheres to $X$. We shall now show that $q(X)$ is an obstruction to a free $S^{1}$ action on the homotopy type of $X$ having quotient space finitely dominated.

THEOREM 3.1. Let $X$ be as above. Suppose there is a fibration $S^{1} \rightarrow X \rightarrow Y$, orientable, with $Y$ of finite type. Then $q(X)=1 \in \mathbf{Q}_{+}^{*}$.

PrOOF. It is easy to check that the space $Y$ is nilpotent. Consider the rationalization of the fibration $S_{(0)}^{1} \rightarrow X_{(0)} \rightarrow Y_{(0)}$. A spectral sequence argument shows that

$$
Y_{(0)}=\mathbf{C} P_{(0)}^{n} \times \prod S_{(0)}^{2 k_{i}+1}
$$

Using obstruction theory (as in $\S 2$ ), we produce a map

$$
\mathrm{C} P^{n} \times \prod S^{2 k_{i}+1} \rightarrow Y
$$

which is a rational homotopy equivalence. The $S^{1}$-fibration $S^{\mathbf{1}} \rightarrow X \rightarrow Y$ is classified by $B S G(2)=B S O(2)=\mathrm{C} P^{\infty}$, and so is given by a 2-dimensional cohomology class. Pulled back to $\mathbf{C} P^{n} \times \prod S^{2 k_{i}+1}$, this must be the 2-dimensional generator of $\mathrm{H}^{2}\left(\mathbf{C} P^{n}\right)$.

Hence we obtain a pullback diagram:

$$
\begin{array}{ccccc} 
& & S^{1} & & S^{1} \\
S^{2 n-1} \times \prod S^{2 k_{i}+1} & \stackrel{g}{\rightarrow} & S^{2 n-1} /(\mathrm{Z} / k) \times \prod S^{2 k_{i}+1} & \stackrel{f}{\rightarrow} & \begin{array}{l}
\downarrow \\
\\
\end{array} \\
& & & & \\
& \mathrm{C} P^{n} \times \prod S^{2 k_{i}+1} & \stackrel{h}{\rightarrow} & Y
\end{array}
$$

Now from $\S 2$ we know that $q(X)=m \chi(f \cdot g)=m \chi(f) \cdot m \chi(g)$. It is easy to see that $m \chi(g)=1$, so we need only compute $m \chi(f)$.

The Serre spectral sequence to compute $H_{*}(f ; \mathbf{Z})$ has

$$
E_{p, q}^{2}=H_{p}\left(S^{1} ; H_{q}(h)\right) \text {. }
$$

Thinking of $E_{*, *}^{2}$ as singly graded by total degree, it is clear that $m \chi\left(E_{*, *}^{2}\right)=1$, since $E_{0, *}^{2}=E_{1, *}^{2}$ and $E_{s, *}^{2}=0$ for $s \neq 0,1$. Also, by a standard argument, the Euler characteristic $m \chi$ does not change by taking homology. Hence,

$$
m \chi\left(E_{*, *}^{2}\right)=m \chi\left(E_{*, *}^{3}\right)=m \chi\left(E_{*, *}^{\infty}\right) \text {. }
$$

But $E_{*, *}^{\infty}$ is the associated bigraded object for the Serre filtering of the graded module $H_{*}(f)$. Hence $m \chi\left(H_{*}(f)\right)=m \chi\left(E_{*, *}^{\infty}\right)=1$.

This of course implies $q(G)=1$ for any Lie group. It is an interesting question whether this is true for any $H$-space. Notice this may also be asked $p$-locally.

4. Linking numbers. In one special situation the invariant $q(X)$ turns out to coincide with a well-known classical invariant.

Let $S_{1}^{n}, S_{2}^{k}$, and $S_{3}^{n+k+1}$ be simply connected oriented manifolds having the rational homotopy type of spheres. 
Suppose we have imbeddings

$$
S_{1}^{n} \underset{i_{1}}{\rightarrow} S_{3}^{n+k+1} \underset{i_{2}}{\leftarrow} S_{2}^{k}
$$

such that $i_{1}\left(S_{1}\right) \cap i_{2}\left(S_{2}\right)=\varnothing$.

Now consider the following diagram:

$$
\begin{array}{rlc}
0 \rightarrow H_{k+1}\left(S_{3}, S_{3}-S_{1}\right) & \stackrel{\partial}{\rightarrow} & H_{k}\left(S_{3}-S_{1}\right) \rightarrow H_{k}\left(S_{3}\right) \\
\mathbf{Z} \cdot\left[S_{1}^{n}\right] \cong H_{n}\left(S_{1}\right) & & H_{k}\left(S_{2}\right) \cong \mathbf{Z} \cdot\left[S_{2}^{k}\right]
\end{array}
$$

We have $H_{k}\left(S_{3}\right) \oplus \mathbf{Q}=0$. Hence there are integers $r, s \in \mathbf{Z}$ such that

$$
s \cdot i_{2^{*}}\left[s_{2}^{k}\right]=r \cdot \partial\left[S_{1}^{n}\right] .
$$

Define the linking number $l\left(S_{3}: S_{1}, S_{2}\right)$ of $S_{1}$ and $S_{2}$ in $S_{3}$ to be

$$
l\left(S_{3} ; S_{1}, S_{2}\right)=r / s \text {. }
$$

This generalizes the usual notion of linking numbers of spheres in spheres. We say that $S_{1}$ and $S_{2}$ are nontrivially linked in $S_{3}$ if $l\left(S_{3} ; S_{1}, S_{2}\right) \neq 0$. For each of the above manifolds we have the degree one map $\alpha_{i}: S_{i}^{s} \rightarrow S^{s}$. Let

$$
M\left(S_{i}^{s}\right)=m_{x}\left(\alpha_{i}\right)=\frac{\prod \mid \text { Tors } H_{\text {odd }}\left(S_{i}\right) \mid}{\prod \mid \text { Tors } H_{\text {even }}\left(S_{i}\right) \mid} .
$$

If $S_{1}$ and $S_{2}$ are nontrivially linked in $S_{3}$, then it is easy to check that the inclusion $S_{1}^{n} \rightarrow S_{3}^{n+k+1}-S_{2}^{k}$ is a rational homotopy equivalence and hence $X=S_{3}-S_{1}-S_{2}$ is rationally $S^{n} \times S^{k}$.

PROPOSITION 4.1. Let $S_{1}^{n}, S_{2}^{k} \subseteq b S_{3}^{n+k+1}$ be rational homology spheres in $s$. Let $X=S^{n+k+1}-S_{1}^{n}-S_{2}^{k}$. If $n$ and $k$ are odd we have

$$
q(x)=\frac{M\left(S_{3}\right)}{M\left(S_{1}\right) \cdot M\left(S_{2}\right)} \cdot l\left(S_{3} ; S_{1}, S_{2}\right) .
$$

In particular if all rational homotopy spheres are genuine spheres our invariant is just the classical linking number.

PROOF. Let $r_{1} \downarrow s_{1}$ be the normal bundle to $S_{1}$ in $S_{3}$. Consider the inclusion $j_{1}: S r_{1} \rightarrow X$. A straightforward calculation shows

$$
m_{x}\left(j_{1}\right)=\frac{M\left(S_{3}\right)}{M\left(S_{1}\right) \cdot M\left(S_{2}\right)} \cdot l\left(S_{3} ; S_{1}, S_{2}\right) .
$$

Let $\tilde{j}_{2}: S^{n} \rightarrow S_{1}^{n}$ be a map of nonzero degree (such a map exists since $S_{1}^{n}$ is a rational homology sphere). This induces

$$
j_{2}: \tilde{j}_{2}^{*}\left(S r_{1}\right) \rightarrow S r_{1}
$$

A spectral sequence argument as in $\S 3$ shows $m_{x}\left(j_{2}\right)=1$. In analogy to [M1] we see that $S r_{1} \downarrow S_{1}$ is rationally fiber homotopically trivial. Hence there is a map

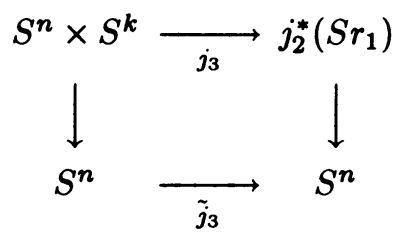


induced by a nonzero degree map $\tilde{j}_{3}$. As before we have $m_{x}\left(j_{3}\right)=1$.

From $\S 2$ we know

$$
\begin{aligned}
q(x) & =m_{x}\left(j_{3} \cdot j_{2} \cdot j_{1}\right)=m_{x}\left(j_{1}\right) \cdot 1 \cdot 1 \\
& =\frac{M\left(S_{3}\right)}{M\left(S_{1}\right) \cdot M\left(S_{2}\right)} \cdot l\left(S_{3} ; S_{1}, S_{2}\right) .
\end{aligned}
$$

\section{BIBLIOGRAPHY}

[M1] W. Massey, On the normal bundle of a sphere imbedded in Euclidean space, Proc. Amer. Math. Soc. 10 (1959), 959-964.

[M2] G. Mislin, Finitely dominated nilpotent spaces, Ann. of Math. (2) 103 (1976), 547-556.

DEPARTMENT OF MATHEMATICS, INDIANA UNIVERSity, BLOOMINGTON, INDIANA 47405

DEPARTMENT OF MATHEMATICS, MATHEMATICHES INSTITÜT DER UNIVERSITÄT, 3400 GÖTTINGEN, WEST GERMANY

MAThematical INSTitute, Odense UNIVERSity, OdENSE DK5000, DENMARK 